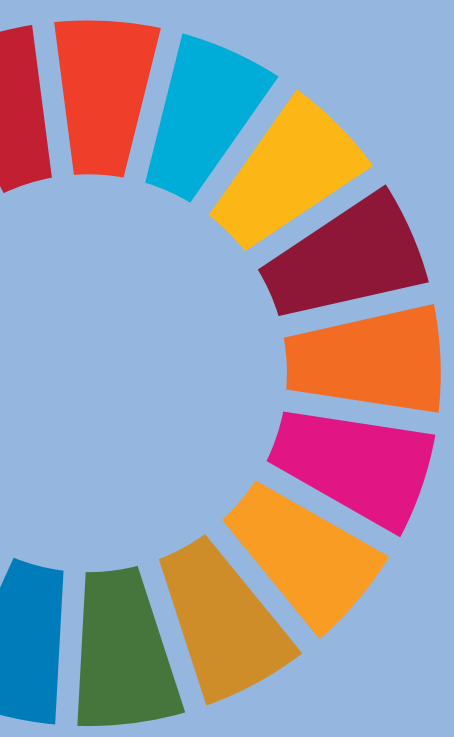

\title{
HACIA UNA NUEVA
}

NORMALIDAD:

\section{OPORTUNIDADES DE}

\section{LA SALIDA DE LA CRISIS}

Y SU CONEXIÓN CON

ASPECTOS AMBIENTALES

\section{TOWARDS A NEW}

\section{REALITY: OPPORTUNITIES}

\section{FOR A CRISIS OUTPUT}

AND ITS RELATIONSHIP

WITH ENVIRONMENTAL

\section{ASPECTS}

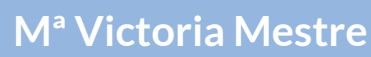

Consejo Económico y Social

victoria.mestre@telefonica.net

Anna Traveset

Consejo Superior de Investigaciones Científicas del Instituto Mediterráneo de Estudios Avanzados

(IMEDEA, CSIC-UIB)

atraveset@imedea.uib-csic.es

Nancy Villaluenga

Ecoembes

n.villaluenga@ecoembes.com

Carmen Recio

Fundación Iberdrola España

creciom@iberdrola.es 


\section{RESUMEN}

Este artículo aborda la coyuntura de algunos aspectos ambientales que, como en el resto del mundo, en España se han visto en mayor o menor medida afectados como consecuencia de las medidas de confinamiento de la población y reducción de la actividad económica registradas durante la crisis de la COVID-19. Se destacan la reducción de las emisiones contaminantes a la atmósfera, la protección del agua como recurso esencial para la vida o el refuerzo de las operaciones destinadas a gestionar los residuos (en especial los de origen sanitario), todos ellos condicionantes de las necesarias garantías de salud de la población. La importancia de la preservación de los recursos naturales revelada durante el Estado de Alarma declarado en estos primeros meses de 2020, debe interpretarse en términos de oportunidad y aprovecharse para impulsar el enfoque sostenible de los sectores que afectan o son afectados por los mismos, tales como el transporte, la depuración y distribución del agua potable o la gestión de los residuos. Para terminar, se aborda brevemente la situación del sector agroalimentario, considerado estratégico durante la crisis sanitaria.

Palabras clave: Contaminación atmosférica, Energía renovable, Calidad del agua, Gestión de residuos, Agricultura sostenible.

\section{ABSTRACT}

This article approaches some environmental aspects which, in Spain as well as in the rest of the world, have been affected, in certain extent, as a consequence of population confinement and economical activity reduction measures, applied during the COVID-19 crisis. Outstands the atmospheric polluting emissions reduction, the water protection as a main resource for life, or the strengthening of measures aimed to waste management (specially those with a sanitary origin), all of them essentials for the necessary guarantees of people's health and safety. The importance of natural resources preservation shown during the State of Alarm declared in early 2020, must be interpreted in terms of opportunity, and promote a sustainable approach in sectors which can affect or be affected by those environmental aspects, such as transport, supply of water and sewage networks, or waste management. Finally, is briefly addressed the agrifood industry situation, considered as a strategic sector during this sanitary crisis.

Keywords: Atmospheric pollution, Renewable energy, Water quality, Waste management, Sustainable agriculture.

Las autoras de este artículo son miembros de la Comunidad de Medio Ambiente y Salud de la plataforma "El día Después será...". Se trata de un grupo de conocimiento multidisciplinar y de práctica que surgió durante el confinamiento y que tiene como objetivo generar acciones concretas para alinear la salida de la crisis de la COVID-19 con la Agenda 2030 de Desarrollo Sostenible, en particular en el ámbito del cuidado de la biodiversidad como medida de protección frente a futuras pandemias.

$M^{a}$ Victoria Mestre es licenciada en CC. Biológicas esp. Medio Ambiente, y doctora en Economía (Programa interuniversitario Economía y Gestión de la Innovación, reconocido por las Universidades Autónoma, Complutense y Politécnica de Madrid). Actualmente forma parte del Área de Estudios y Análisis del Consejo Económico y Social, y se ocupa de aspectos relacionados con Agenda 2030 y ODS, cambio climático, economía circular y bioeconomía. Con anterioridad ha participado en equipos de evaluación de impacto ambiental en la empresa privada, y de educación ambiental para la Comunidad de Madrid. 
Anna Traveset es Profesora de Investigación del Consejo Superior de Investigaciones Científicas del Instituto Mediterráneo de Estudios Avanzados (IMEDEA, CSIC-UIB), con sede en Mallorca. Recibió su Licenciatura en Biología en la Universidad de Barcelona y realizó su doctorado en la Universidad de Pennsylvania, Philadelphia (USA). Su investigación se centra principalmente en sistemas insulares, aunque también colabora en proyectos en el continente, explorando cómo las interacciones bióticas influyen en la regeneración y distribución de las plantas, y cómo dichas interacciones son influenciadas por distintos motores de cambio global.

Nancy Villaluenga es licenciada en Ciencias Económicas por la Universidad Complutense de Madrid, con Máster en Ecoauditorías y Planificación Empresarial del Medio Ambiente por el Instituto de Investigaciones Ecológicas. Actualmente, es coordinadora de Relaciones Institucionales en Ecoembes, organización sin ánimo de lucro dedicada al reciclaje y el ecodiseño de los envases domésticos en España. Su trabajo está centrado en el impulso de alianzas con organizaciones sociales y ambientales, a fin de promover y fortalecer iniciativas para la protección del medio ambiente.

Carmen Recio es Licenciada en Ciencias Económicas y Empresariales por la Universidad Complutense de Madrid. Máster en Organización Jurídica, Económica y Social del Medio Ambiente por la Escuela de Organización Industrial, Curso Superior de Finanzas de la Energía: Energía y Proyectos por la Universidad de Alcalá de Henares/ GIFF y I Edición del Programa Superior de Dirección en Responsabilidad Corporativa por el Instituto de Empresa, Madrid. Ha sido vocal del Consejo Estatal de Responsabilidad Social Empresarial (dependiente del Ministerio de Trabajo) desde 2009 a 2014. En la actualidad, forma parte del equipo de la Fundación Iberdrola España como Responsable de las Áreas de Biodiversidad y Cambio Climático y de Arte y Cultura.

\section{INTRODUCCIÓN}

La emergencia ocasionada por la COVID-19 en marzo de 2020, elevada a pandemia por la Organización Mundial de la Salud (OMS), ha provocado una crisis sanitaria y socioeconómica sin precedentes en la historia reciente española y europea.

Las medidas de restricción de la movilidad y la actividad económica han repercutido en el medio ambiente desde diversas perspectivas. Junto a los efectos inmediatos de la reducción de la contaminación atmosférica sobre la calidad del aire, otros aspectos no tan evidentes pero vinculados a la disponibilidad de recursos naturales -como la dotación de energía a los hogares, el suministro de agua potable de calidad o la gestión de los residuos y vertidos sometidos a condiciones de garantía de abastecimiento y/o gestión durante el confinamiento de la población- han evidenciado su carácter esencial para la calidad de vida. El aumento de la vegetación o la presencia de especies de fauna antes no vistas en espacios urbanos se destacan entre los efectos más perceptibles de la mejora ambiental asociada a una menor presión de la actividad humana, si bien se espera que estos efectos positivos sean coyunturales, en ausencia de políticas que favorezcan un cambio estructural en el modelo de desarrollo.

A partir de los resultados del análisis de los impactos asociados a las medidas aplicadas durante la gestión de la crisis sanitaria, este artículo resume la coyuntura de algunos factores que se han visto afectados durante la crisis de la COVID-19, así como las oportunidades asociadas a la dinamización de los sectores vinculados a determinados factores ambientales. 


\section{MEJORA MOMENTÁNEA DE LA CONTAMINACIÓN ATMOSFÉRICA}

Los contaminantes atmosféricos de mayor presencia en España, como los óxidos de nitrógeno ( $\mathrm{NO}_{\mathrm{x}}$ ), las partículas (en especial las de menor tamaño o $\mathrm{PM}_{2,5}$ ) o el ozono troposférico, pueden aumentar la mortalidad por causas respiratorias y vasculares, y la morbilidad asociada a alteraciones en el funcionamiento pulmonar; pero también son responsables de provocar acidificación y eutrofización de los ecosistemas o alterar el crecimiento de cultivos y bosques al reducir la capacidad de absorción de $\mathrm{CO}_{2}$.

Entre 1990 y 2018 las emisiones en España de $\mathrm{NO}_{x}$ y de Compuestos Orgánicos Volátiles no metánicos (NMVOC) experimentaron caídas del $48 \%$ y $39 \%$ respectivamente, alcanzando el $-23 \%$ respecto a 2000 en el caso de las $\mathrm{PM}_{2,5}$ (Figura 1).

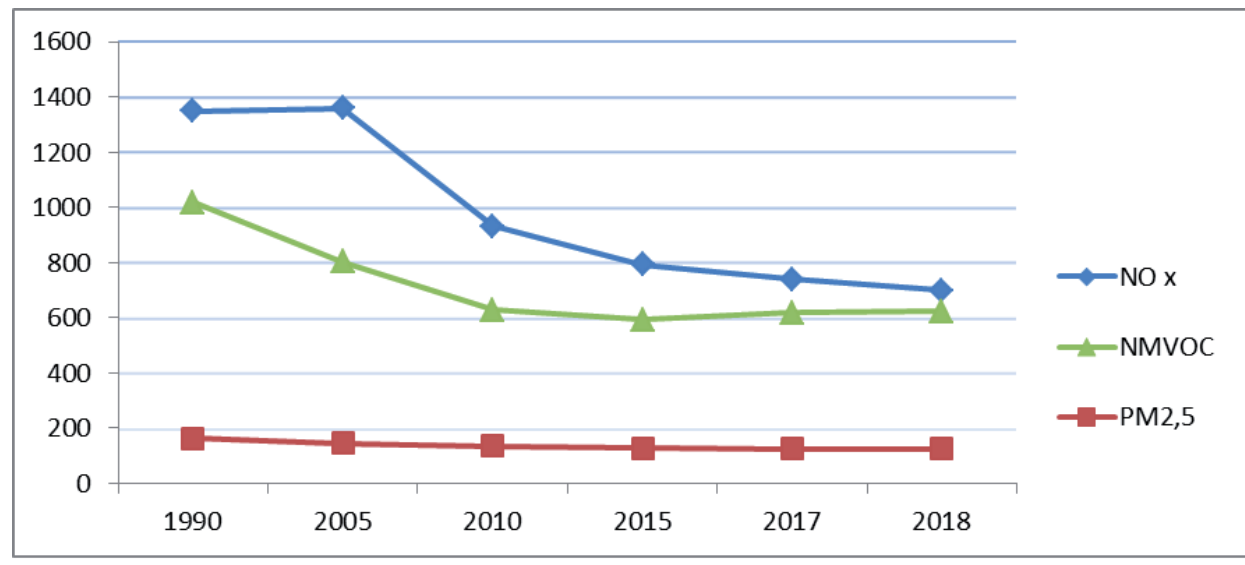

\begin{tabular}{|c|l|c|}
\hline \multicolumn{2}{|c|}{2018} \\
\hline NO $x$ & Transporte & $45,00 \%$ \\
\hline PM2,5 & Fuentes estacionarias combustión & $43,30 \%$ \\
\hline NMVOC & Uso industrial de disolventes & $45,30 \%$ \\
\hline
\end{tabular}

Figura 1. Emisión de principales contaminantes a la atmósfera en España antes de la COVID-19 (Ministerio para la Transición Ecológica y el Reto Demográfico [MITECO], 2019)

A finales del primer trimestre de 2020, las medidas de limitación de la libre circulación de las personas adoptadas como consecuencia de la COVID-19 (Real Decreto 463/2020, de 14 de marzo, que declara el estado de alarma en España), derivaron en la reducción drástica y generalizada del transporte, la actividad industrial y la generación de electricidad. Como resultado, el tráfico urbano e interurbano, así como el acceso a las principales ciudades se redujeron en torno al $70 \%$, mientras el traslado de productos petrolíferos desde las instalaciones del Grupo CLH (Compañía Logística de Hidrocarburos) al mercado español descendió un $75 \%$ en el caso de la gasolina, un $55 \%$ en el caso del gasóleo A y un $93 \%$ en el de los carburantes de aviación. La demanda eléctrica también cayó un $20 \%$ respecto a la situación previa a las medidas de restricción (Ecologistas en Acción [EeA], 2020).

En Madrid y Barcelona, las estaciones en las zonas de bajas emisiones (Madrid Central y Rondas de Barcelona) experimentaron reducciones de los niveles de $\mathrm{NO}_{2}$ del $60 \%$ y el $62 \%$ respectivamente, muy superiores a las resultantes de la aplicación de medidas de restricción del tráfico motorizado previas a la crisis. De hecho, los niveles de $\mathrm{NO}_{2}$ registrados durante el estado de alarma fueron los más bajos de la última década. Se ha apreciado también una caída significativa de los niveles de partículas PM $_{10}$ 
y $\mathrm{PM}_{2,5}$, así como de la concentración de ozono en España en el inicio de la primavera, en zonas tanto urbanas como rurales. La reducción de las emisiones de $\mathrm{NO}_{x}$ y de NMVOC, principales precursores del ozono, junto a la meteorología inestable y lluviosa responsable de una menor radiación solar (necesaria para la formación del ozono), podrían explicar esta circunstancia. Así pues, el impacto positivo generado por las medidas de la COVID-19 sobre la atmósfera se añade a la tendencia positiva que ya se venía registrando.

Algunas investigaciones subrayan la oportunidad que representa la situación generada para mantener una adecuada calidad del aire durante la desescalada y en la época post COVID-19, en ámbitos tan diversos como:

- La mejora de la planificación territorial y promoción urbanística, que debe poner en relación los núcleos residenciales con el empleo y los servicios, y planificar el transporte público. Las tecnologías de información y la comunicación (TICS), pueden contribuir a optimizar los desplazamientos y la logística.

- El impulso a la adquisición de vehículos limpios y energéticamente eficientes, así como a la movilidad eléctrica, con impacto positivo en el sector de la automoción.

- La aparición de nuevos modelos de negocio que oferten servicios de transporte flexibles y competitivos, basados en la electromovilidad, la movilidad compartida (Mobility as Service, MaaS) y la digitalización, que transformen progresivamente la demanda de los usuarios.

- La gestión del transporte en el espacio urbano, optimizando la demanda mediante el fomento del teletrabajo, la flexibilización de horarios para evitar congestión en horas punta, la creación de zonas verdes, el impulso a la intermodalidad y la adecuación del transporte público (European Environment Agency [EEA], 2019).

\section{LAS RENOVABLES SE ABREN CAMINO}

Más de la mitad del consumo de energía final en España correspondió en 2018 al petróleo (52,8\%), respondiendo el resto de la demanda a la electricidad (22\%), el gas natural (16,5\%) y el carbón (1,9\%), cuotas que reflejan una todavía elevada dependencia energética, que ese año alcanzó el 73,4\% (Asociación Española de Energía Renovable [APPA], 2018). En 2019, la generación de electricidad en España fue en un $36,8 \%$ renovable, y en un 58,6\% libre de $\mathrm{CO}_{2}$ (incluyendo la de origen nuclear), siendo el ciclo combinado la primera fuente de generación (21,9\%), seguida de la nuclear $(21,2 \%)$ y de la eólica $(20,6 \%)$ (Red Eléctrica Española [REE], 2019).

Durante el estado de alarma declarado en 2020 , la demanda de energía eléctrica se redujo un 17,6\% respecto a semanas anteriores, lo que se atribuye a un descenso de la actividad económica que afectó principalmente a los sectores terciario e industrial, mientras el aumento registrado en la demanda doméstica debido al confinamiento no compensaba la caída de la demanda industrial. Consecuentemente, la emisión media de $\mathrm{CO}_{2}$ asociada a la generación eléctrica se redujo entre el 15 de marzo y el 27 de abril un 6,3\% respecto al mes anterior, escaso si se compara con la fuerte reducción de la demanda, lo que parece estar relacionado con el aumento de la participación de ciclos combinados de gas en la generación eléctrica (Greenpeace, 2020). 
El impulso a las energías renovables pasa por optimizar la gestión de la demanda, especialmente en situaciones de crisis como la generada por la pandemia, en las que es necesario aumentar la seguridad del suministro desplazando los picos de demanda a los momentos de máxima generación de energía, o aumentando la eficiencia y evitando consumos innecesarios. Además, debería abordarse una reforma en el diseño de la facturación, para que la bajada de precios asociada al aumento de renovables o al descenso de la demanda llegue tanto a los mercados mayoristas como al consumidor. Por otro lado, convendría reducir el uso especulativo de energía hidráulica, que aumenta su generación en los momentos de mayores precios marginales (Greenpeace, 2020).

También en este caso se abre un escenario de oportunidades asociado al objetivo de alcanzar al menos un 70\% de electricidad renovable para 2030 establecido en el Proyecto de Ley de Cambio Climático y Transición Energética:

- Despliegue de medios técnicos y financieros asociados a la instalación anual de unos 3.000 MW de origen renovable que sustituya al carbón y las nucleares hacia 2030 (MITECO, 2020);

- Mejora de la interconexión eléctrica con el resto de las redes europeas para equilibrar, cuando sea necesario, los picos de demanda y oferta de las renovables;

- Fomento de la investigación orientada a resolver los problemas de almacenamiento de la electricidad a partir de renovables, con el desarrollo de baterías de nueva generación, hasta ahora mayoritariamente importadas.

- Difusión y concienciación ciudadana sobre el estado de la ley de autoconsumo, que ha sido impulsado por el RD de Autoconsumo 244/2019 y el RDL 15/2018. Entre notables cambios, ha introducido la posibilidad de instalar autoconsumo compartido en bloques de viviendas y el mecanismo de compensación simplificada, a través del cual los autoconsumidores pueden recibir una compensación económica para los excedentes de energía que vierten a la red (Unión Española Fotovoltaica [UNEF], 2020).

Todo ello requiere el desarrollo de un marco regulador eficaz que ofrezca seguridad jurídica, impulse la inversión, y mejore la competitividad del sector, evitando comportamientos especulativos.

\section{OPTIMIZAR LA RED DE ABASTECIMIENTO Y DEPURACIÓN DE AGUA}

El agua es un recurso esencial para la vida y deficitario en todo el mundo. Especialmente en el caso de España, donde la meteorología diversa y desigual distribución de los recursos hídricos, junto a los efectos del cambio climático, provocan una situación de déficit que afecta su disponibilidad y calidad al reducir el oxígeno disuelto, provocando una merma en la capacidad de autopurificación de las cuencas de agua dulce, y aumentando el riesgo de contaminación o la presencia de patógenos.

En España, el 67\% del consumo de agua se destina al sector agrario, un 19\% al industrial y un 14\% a usos urbanos (Price Waterhouse Coopers [PWC], 2018). El suministro a la red de abastecimiento público supone en España unos 4,3 $\mathrm{Hm}^{3}$, de los que el 74,6\% está registrada (AR) en medidores de los clientes, mientras el resto, definido como agua no registrada (ANR), se distribuye entre consumos no medidos y pérdidas en la red. Pese a la mejora desde el año 2000, estas últimas representan un 16,3\% del total de agua suministrada. La 
situación actual es de cierto envejecimiento de las redes de distribución asociado a un déficit en la inversión, ya que el $39 \%$ cuenta con más de 30 años, el $36 \%$ más de 10 años y sólo el $17 \%$ restante menos de 10 . Lo mismo ocurre con las de alcantarillado, de las que el $58 \%$ tiene más de 30 años, el $31 \%$ más de 10 años y el 11\% restante menos de 10 (Asociación Española de Abastecimiento de Agua y Saneamiento [iAgua], 2018) (Figura 2).

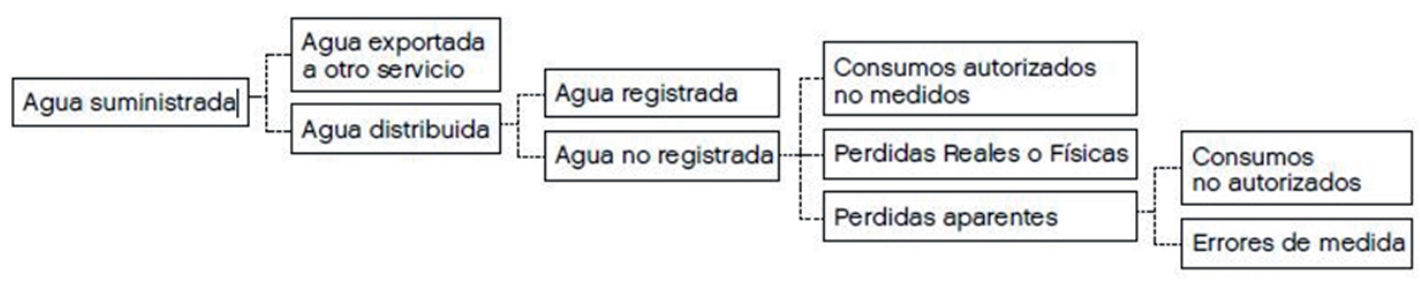

Pérdidas en la red de abastecimiento público

(\% sobre agua suministrada)

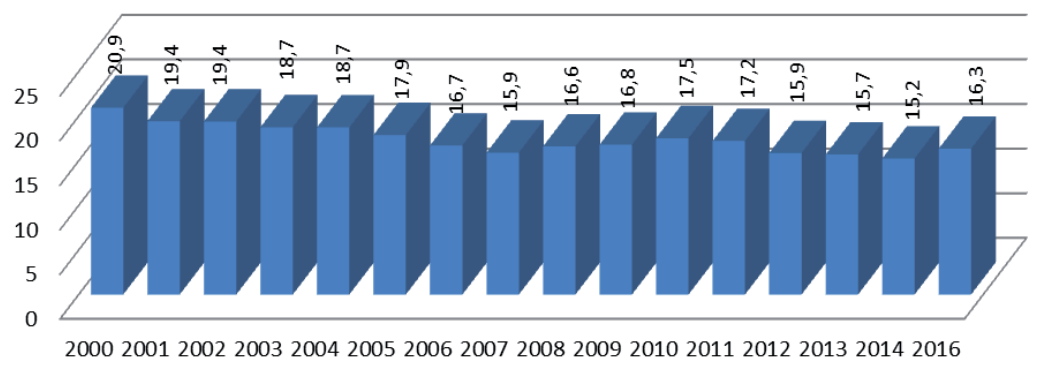

Figura 2: Esquema de distribución del agua suministrada (iAgua, 2018). Pérdidas en la red de abastecimiento público (\% sobre agua suministrada) (Instituto Nacional de Estadística [INE], 2020).

Tanto el suministro de agua como el tratamiento de aguas residuales se han considerado esenciales durante el estado de alarma generado por el COVID-19, por lo que ha sido necesario garantizar la calidad de los servicios a los ciudadanos y de seguridad a los empleados (Ministerio de Sanidad, Orden SND/274/2020). La OMS no contempla el agua entre las vías de transmisión del virus, siempre que esté adecuadamente tratada, ya que tanto los tratamientos de potabilización como los de depuración avanzada aseguran su correcta eliminación. Por el contrario, sí se ha detectado la presencia de material genético del virus en aguas residuales no tratadas, es decir, antes del tratamiento de depuración en las estaciones depuradoras de aguas residuales (EDAR), desapareciendo tras los tratamientos de depuración convencionales (Asociación Española de Desalación y Reutilización [AEDyR], 2020).

Todo ello, más aún en el contexto de crisis sanitaria como la actual, refuerza la necesidad y oportunidad de abordar los necesarios cambios en el corto plazo (World Health Organization [WHO], 2020):

- Mejora de la red de abastecimiento y depuración de las aguas, mediante el impulso de la inversión privada, ante la dificultad de financiación pública de la infraestructura hidráulica;

- Optimización de la planificación hidrológica mediante un modelo de gestión que responda en términos de calidad y cantidad a la demanda de este recurso escaso y esencial. 


\section{GESTIÓN DE LOS RESIDUOS: MUCHO POR HACER}

En 2017, se generaron en España 132,1 millones de toneladas de residuos, un 2,3\% más que el año anterior, en su mayoría no peligrosos (97,6\%), correspondiendo en un $82,9 \%$ a los sectores de actividad y en un $17,1 \%$ a los hogares. De los 115,5 millones de toneladas gestionadas (un 8,2\% más que el año anterior), el 53,9\% se destinaron a vertederoy el $38,9 \%$ a reciclaje (Figura 3 ).

Ese año, el 3\% de los residuos correspondían a envases y embalajes, de los que se reciclaron el 68,5\% (Oficina Europea de Estadística [Eurostat], 2020). Las tasas de reciclado de estos materiales han registrado un aumento continuo entre 2006 y 2017, hasta alcanzar casi el $48 \%$ la del plástico, superar el $70 \%$ en los casos del vidrio, el papel y cartón, y el $85 \%$ en el caso de los metales (Figura 4).
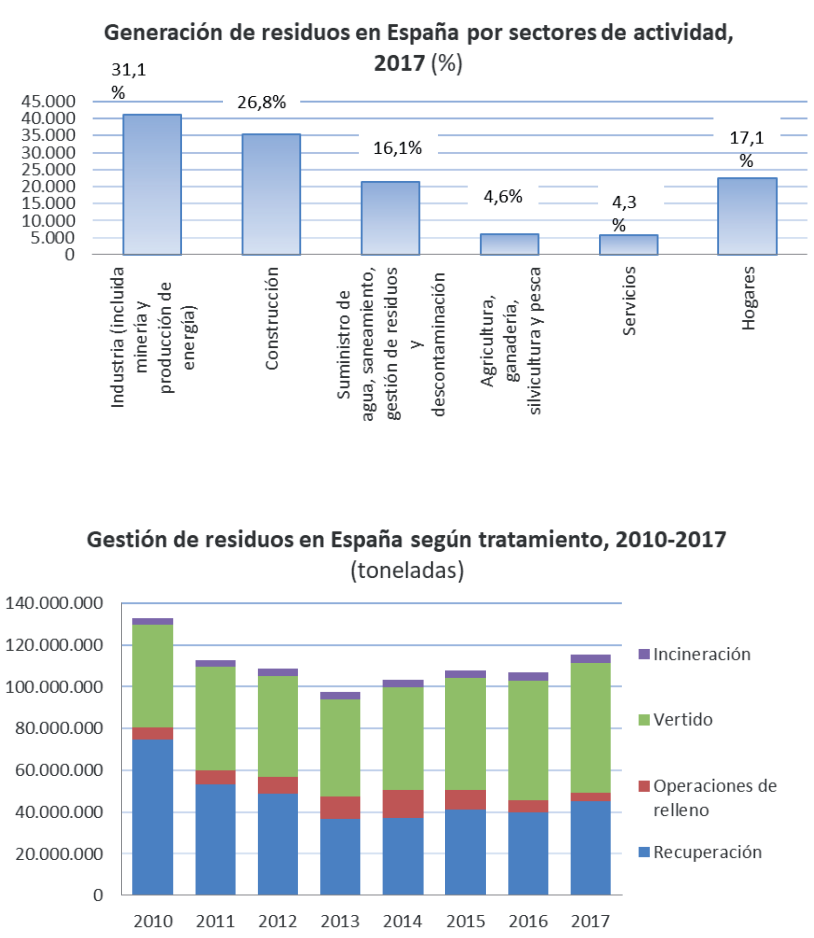

Figura 31: Los residuos en España: generación y gestión (INE, Estadísticas sobre recogida y tratamiento de residuos).

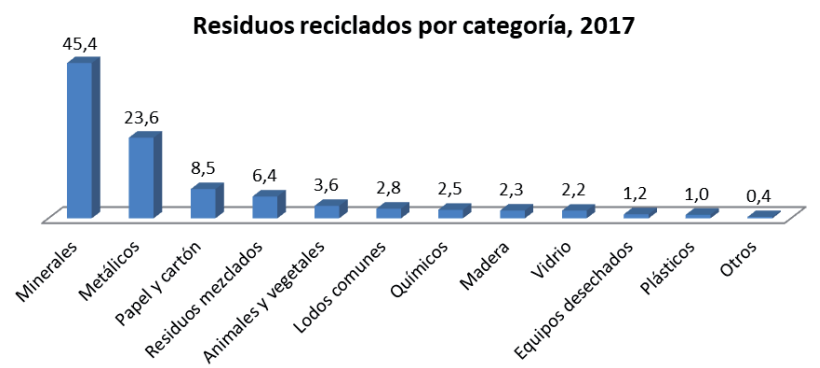

\footnotetext{
1 Nota: las operaciones de recuperación de residuos incluyen operaciones de regeneración, reciclado y compostaje (excluyen las opera-
} ciones de relleno o de recuperación energética). 


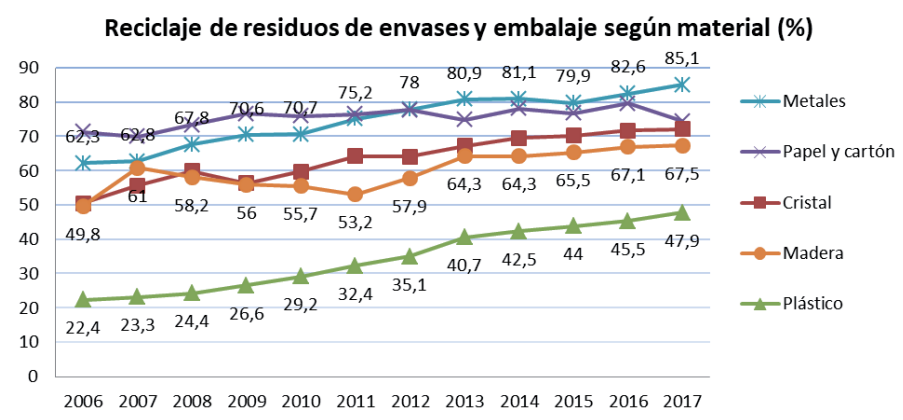

Figura 4. Residuos reciclados por categoría (\%) y Reciclaje de residuos de envases y embalajes (\%) (INE y Eurostat)

Desde el inicio del estado de alarma el 14 de marzo de 2020, los ciudadanos españoles han incrementado en un $15 \%$ el uso del contenedor amarillo para reciclar sus envases de brik, plástico y metal; simultáneamente se ha observado una tendencia a la baja en los residuos depositados tanto en el contenedor azul (papel/ cartón) como en el gris (resto). Ambas caídas responderían al parón de la actividad económica -sobre todo en hostelería y resto de sector comercial y servicios- en todo el país. Según el informe "La sostenibilidad, el compromiso medioambiental y el reciclaje tras la COVID-19", más de 2,5 millones de ciudadanos declaran haber empezado a separar algún nuevo tipo de residuo durante confinamiento. Más allá del compromiso con el reciclaje, es un ejemplo del creciente grado de preocupación por las cuestiones ambientales. De hecho, más de la mitad de los encuestados incluye aspectos como el cambio climático o la contaminación, entre los retos prioritarios de la sociedad post-COVID (Ecoembes, 2020). Los ayuntamientos han garantizado la recogida selectiva de los residuos para su posterior reciclado, priorizando la seguridad de los empleados de las plantas y gestionando de forma automatizada y separada los procedentes de hogares con algún miembro en cuarentena o infectado por la COVID-19, en cumplimiento de las recomendaciones procedentes del Ministerio de Transición Ecológica y Reto Demográfico y la Federación Española de Municipios y Provincias (FEMP). Por otra parte, durante la COVID-19, guantes y mascarillas se han convertido en nuevos protagonistas de la basura dispersa, provocando la activación de múltiples campañas de sensibilización por parte de administraciones públicas, empresas y ONGs.

El calendario propuesto para reducir el peso de los residuos producidos -que se añade al vigente del $10 \%$ en 2020- es del 13\% en 2025, y del 15\% en 2030, respecto a 2010 (en 2017 la generación fue un 7,6\% inferior a la de 2010). Por su parte, la preparación para la reutilización y reciclado de residuos municipales respecto a los generados debe alcanzar el 55\% en 2025, el 60\% en 2030, y el 65\% en 2035 (frente a esas cifras el reciclaje alcanzó en 2017 el 38,9\%). En todo caso, el vertido debe ser la última opción en la gestión de los residuos (MITECO, Anteproyecto de Ley de Residuos y Suelos Contaminados). Hay mucho recorrido para abordar y cumplir unos objetivos que están a punto de implantarse en España. Junto a los 20 sistemas de responsabilidad ampliada del productor ya existentes para residuos como los envases domésticos, los aparatos eléctricos o los neumáticos, se regulará, en función de la obligatoriedad de recogida separada, la previsible creación, en los próximos años, de otros modelos similares para residuos comerciales e industriales, textil, artes de pesca, etc. También será importante el desarrollo de sistemas de pago por generación vinculados a la tasa de basuras, o la universalidad de la recogida selectiva obligatoria en cualquier ámbito público o privado. La transición requiere un marco instrumental y una actividad coordinada entre administraciones, sectores económicos y el conjunto de la sociedad para facilitar sinergias. 
En la consecución de los objetivos propuestos, es necesario:

- Desarrollar y aplicar conocimientos y capacidades que den lugar a nuevos desarrollos tecnológicos, procesos, productos y servicios innovadores que contribuyan a la competitividad empresarial, generen oportunidades de negocio y nuevas cadenas de valor, e impulsen la creación de empleo.

- Adoptar innovaciones tecnológicas, organizativas y sociales, para generar el cambio en los modelos de producción y consumo, transformando, cuando sea preciso, la venta de productos en prestación de servicios (MITECO, España Circular 2030).

- Implementar un sistema de acreditación oficial de los datos en materia de residuos que clarifique los datos de partida, audite las estadísticas y coordine la información adecuadamente para incentivar la separación de residuos, el consumo responsable y la prevención de la basura dispersa, así como para fortalecer la confianza y la participación ciudadana.

- Promover la educación ambiental como herramienta clave para la integración de los principios de circularidad en la sociedad.

\section{HACIA UNA AGRICULTURA CLAVE Y SOSTENIBLE}

El sector agroalimentario es clave para la economía española, al generar, junto con la silvicultura y la pesca, el 2,8\% del PIB nacional; y es esencial porque es la base sobre la que se sustentan más de 30.700 industrias agroalimentarias que, junto a la industria auxiliar de fertilizantes y semillas, el transporte y la distribución alimentaria, aportan cerca del 11\% del PIB nacional y generan 2,7 millones de empleos en España.

Más allá de su papel clave en la economía, el sector ha mantenido un aumento de las prácticas sostenibles hasta alcanzar en 2018 los 2,24 millones de hectáreas, el 9,3\% del total del Área Agrícola Utilizada en España, ocupando el primer lugar en la Unión Europea en superficie dedicada a agricultura ecológica, con el 16,7\% sobre el total (Figura 5).

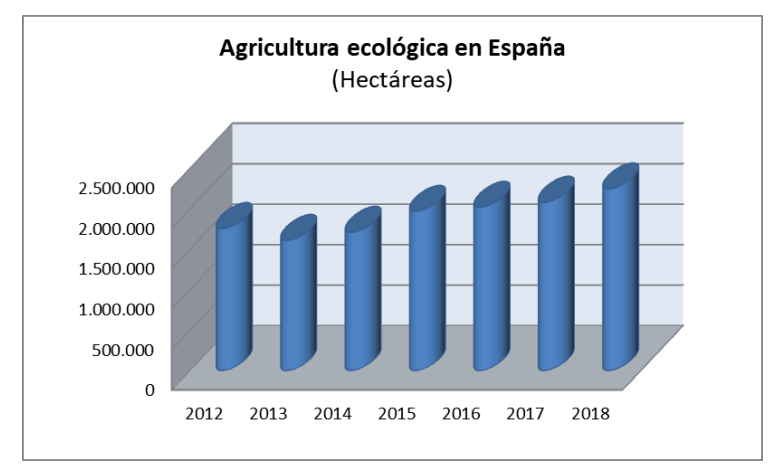

Figura 5. Agricultura ecológica en España (Eurostat). 
Durante la crisis de la COVID-19 el sector ha sido considerado estratégico, conforme al artículo 15 del Real Decreto 463/2020, que declara el estado de alarma. En su cumplimiento se ha garantizado el comercio minorista asociado a la actividad agropesquera, y el que suministra insumos al sector (semillas, fertilizantes, hielo, etc.), la producción agrícola, ganadera y acuícola, la actividad pesquera, la transformación de productos agrarios y pesqueros, los centros o clínicas veterinarias, el transporte y distribución de alimentos, así como su comercialización a través de la venta minorista al consumidor, que forman la cadena de abastecimiento alimentario cuya actividad ha debido garantizarse en la situación de estado de alarma (Ministerio de Agricultura, Pesca y Alimentación [MAPA], 2020). El sector agroalimentario español ha demostrado durante la crisis de la COVID-19 su capacidad para seguir funcionando con eficacia en circunstancias excepcionales, manteniéndose la actividad agraria así como los intercambios entre productores, industrias y comercio (mayorista y minorista).

Junto a la cuestión comercial (precios justos, venta a pérdidas, etc.), que exige un tratamiento político transversal, otras cuestiones relacionadas con el sector productor siguen pendientes de solución y continuarán formando parte de la agenda política después de la pandemia. Las oportunidades abiertas se refieren concretamente a:

- El papel de las nuevas generaciones, decisivo en el reto de la digitalización, tanto en la incorporación de nuevas tecnologías, como en la apuesta por estrategias comerciales innovadoras (incluidos los mercados de proximidad) y modelos de vertebración más eficientes.

- El uso eficiente del regadío, que precisa de un impulso a las tecnologías digitales para avanzar hacia una agricultura de precisión que facilite el ahorro de agua y aproveche los insumos.

- El impulso al comercio electrónico rural, que podría ser una solución para numerosos municipios dedicados a la agricultura y a la ganadería. Se potenciarían así tanto los Productos de $\mathrm{Km} 0$, como los productos orgánicos o naturales. Además, la eliminación de intermediarios aumentaría los márgenes de precios, que estarían marcados por el mercado (Moyano Estrada, 2020).

- El impulso financiero, que permita disponer de una red de explotaciones agrarias, en condiciones de ser productivas, y una cadena alimentaria organizada con eficacia (Plan Estratégico Nacional PAC post-2020).

\section{CONCLUSIONES}

Las medidas de restricción de la movilidad y la actividad económica aplicadas durante la crisis sanitaria provocada por la COVID-19 en los primeros meses de 2020 han repercutido en el medio ambiente desde diversas perspectivas. Junto a los efectos más visibles de reducción de la contaminación atmosférica, otros aspectos como la dotación de energía a los hogares, el suministro de agua potable de calidad, la gestión de los residuos o el sector agroalimentario han evidenciado su carácter esencial para la calidad de vida de las personas. Ello ha llevado a analizar los retos y oportunidades asociados al desarrollo de las energías renovables, a las redes de distribución y la depuración de agua en los núcleos urbanos, a la generación y gestión de los residuos municipales o a la producción de alimentos en España.

El potencial de mejora en todos estos aspectos es elevado en España, y urgente la puesta en marcha de medidas orientadas a su optimización y a un desempeño más sostenible, de las que en buena parte dependerá disponer de una mejor capacidad preventiva y protectora de la salud y el medio ambiente frente a posibles futuros episodios de naturaleza epidémica. 


\section{REFERENCIAS BIBLIOGRÁFICAS}

- Asociación Española de Abastecimiento de Agua y Saneamiento. (2018). XV Estudio Nacional de Suministro de Agua Potable y Saneamiento en España 2018.

- Asociación Española de Desalación y Reutilización. (05 de mayo del 2020). Coronavirus, agua potable y aguas residuales (Revista Aguas Residuales).

- Asociación Española de Energía Renovable. (2018). Estudio del Impacto Macroeconómico de las Energías Renovables en España 2018.

- Ecologistas en Acción. (2020). Propuestas para una desescalada con aire limpio. Efectos de la crisis de la COVID-19 en la calidad del aire urbano en España. Resultados provisionales a 30 de abril de 2020 para las 26 principales ciudades.

- European Environment Agency. (2019). The european environment state and outlook 2020. Knowledge for transition to a sustainable Europe.

- Greenpeace. (2020). Análisis y seguimiento del comportamiento del mercado eléctrico-estado de alarma COVID-19.

- Instituto Nacional de Estadística. Cuenta de los residuos 2017.

- Instituto Nacional de Estadística. Estación depuradora de aguas residuales

- Instituto Nacional de Estadística. (2020). Estadísticas sobre recogida y tratamiento de residuos.

- Ministerio de Agricultura, Pesca y Alimentación. (2020). Guía Práctica del sector agrario y pesquero. COVID-2019.

- Ministerio de Sanidad, Orden SND/274/2020 de 22 de marzo 2020, por la que se adoptan medidas en relación con los servicios de abastecimiento de agua de consumo humano y de saneamiento de aguas residuales.

- Ministerio para la Transición Ecológica y el Reto Demográfico. Anteproyecto de Ley de Residuos y Suelos Contaminados.

- Ministerio para la Transición Ecológica y el Reto Demográfico. (2020). Plan Nacional Integrado de Energía y Clima (PNIEC) 2021-2030.

- Ministerio para la Transición Ecológica y el Reto Demográfico. (Consejo de Ministros 02.06.2020). Estrategia Española de Economía Circular. España Circular 2030.

- Ministerio para la Transición Ecológica y el Reto Demográfico. (2019). Informe sobre el Sistema Español de Inventario (1990-2018).

- Ministerio para la Transición Ecológica y el Reto Demográfico. (2019). Informe de Evaluación de la Calidad del Aire en España 2018.

- Moyano Estrada, E. (08 de mayo de 2020). La Agricultura española: más allá del Coronavirus (IESA-CSIC).

- Oficina Europea de Estadística. (2020). Statistics on Organic crop area by agricultural production methods and crops.

- Oficina Europea de Estadística. (2020). Statistics on Recycling rates for packaging waste.

- Price Waterhouse Coopers. (2018). La gestión del agua en España. Análisis y retos del ciclo urbano del agua.

- Red Eléctrica Española. (2019). España cierra 2019 con un 10\% más de potencia instalada de generación renovable (Nota de Prensa 19.12.2019). 
- Unión Española Fotovoltaica (2020). La nueva regulación permite el despliegue del autoconsumo en España (Nota de prensa, 05 de mayo de 2020).

- World Health Organization. (2020). Strengthening Preparedness for COVID-19 in Cities and Urban Settings. 